University of Nebraska - Lincoln

DigitalCommons@University of Nebraska - Lincoln

March 2003

\title{
Consideration of possible mass and velocity corrections to magnetic cluster experiments
}

\author{
Z.Y. Liu \\ University of Nebraska-Lincoln \\ Peter A. Dowben \\ University of Nebraska-Lincoln, pdowben@unl.edu
}

A.P. Popov

Engineering Physics Institute, Moscow State University, Moscow, Russia

David P. Pappas

National Institute of Standards and Technology, Magnetic Recording Measurements, Boulder, Colorado

Follow this and additional works at: https://digitalcommons.unl.edu/physicsdowben

Part of the Physics Commons

Liu, Z.Y.; Dowben, Peter A.; Popov, A.P.; and Pappas, David P., "Consideration of possible mass and velocity corrections to magnetic cluster experiments" (2003). Peter Dowben Publications. 13.

https://digitalcommons.unl.edu/physicsdowben/13

This Article is brought to you for free and open access by the Research Papers in Physics and Astronomy at DigitalCommons@University of Nebraska - Lincoln. It has been accepted for inclusion in Peter Dowben Publications by an authorized administrator of DigitalCommons@University of Nebraska - Lincoln. 


\title{
Consideration of possible mass and velocity corrections to magnetic cluster experiments
}

\author{
Z. Y. Liu and P. A. Dowben \\ Behlen Laboratory of Physics, University of Nebraska, Lincoln, Nebraska 68588-0111 \\ A. P. Popov \\ Engineering Physics Institute, Moscow State University, Kashirshoe Shosse 31, Moscow 115409, Russia \\ David P. Pappas \\ National Institute of Standards and Technology, Division 816.01, Magnetic Recording Measurements, 325 Broadway, \\ Boulder, Colorado 80305-3328
}

(Received 10 January 2002; revised manuscript received 10 July 2002; published 12 March 2003)

\begin{abstract}
Gadolinium occurs, in natural abundance, as several isotopes. The possible combinations of different gadolinium isotopes dictates that even for a fixed number of atoms in the cluster, clusters of gadolinium atoms will exhibit a range of masses. This and the expected consequence of the translation energy distributions are explored as possible corrections to Stern-Gerlach cluster beam-deflection experiments. Upon closer inspection of the experimental data, we find that the translation energy plus the vibrational temperature distribution may be inhomogeneous. This could be the origin of a long tail to high deflections in the experimental deflection profiles, at low cluster temperatures, in the magnetic cluster Stern-Gerlach experiments.
\end{abstract}

DOI: 10.1103/PhysRevA.67.033202

PACS number(s): 36.40.Cg, 31.15.Bs, 34.50.Ez, 71.20.Eh

\section{INTRODUCTION}

Clusters between 2 and 50 atoms are important because they cover the regime between atomic and bulklike properties [1]. In the last decade, the magnetic properties of isolated nanoparticles and clusters have attracted considerable interest. One approach to the study of magnetic clusters has been to undertake Stern-Gerlach cluster beam-deflection experiments [2-8]. In contrast to the case of single atoms, starting from a linear trajectory, the deviation of free magnetic clusters passing through the transverse gradient field in the SternGerlach experiment results in a deflection profile of a certain width and shape. The shape of the deflection profile is different for the transition-metal and rare-earth clusters. In addition, the deflection profile changes with "cluster" temperature and external field.

One of the typical features of the deflection profiles, for a variety of clusters and regimes of the Stern-Gerlach experiment, is the systematic observation of the long tail in the direction of strong field corresponding to the largest deflections. This tail makes the deflection profile asymmetric [7]. Typically, for the $3 d$ transition metals, the individual isolated clusters are characterized as exhibiting superparamagnetic behavior. The effect of the gradient magnetic field is simply to shift the spatial profile of each cluster size toward the direction of strong magnetic field. For isolated clusters of the ferromagnetic rare earths, some new phenomena have been observed [7]. Depending on cluster size (the number of atoms in the cluster), some rare-earth clusters exhibit superparamagnetic behavior, but not all $[2,5]$.

Generally, recent theoretical efforts have been directed towards solving, first, the cluster spin-relaxation problem and only then to describe the deflection profile [9-14]. Within superparamagnetic theory, it is generally assumed that there is small cluster anisotropy energy compared to thermal fluctuations and thus a random distribution of the cluster-spin orientation. The superparamagnetic theory applied to clusters predicts a Gaussian-like functional form of the deflection profile, i.e., generally symmetric with respect to some average deflection [9]. The deviations of the experimental data from the symmetric shape in the deflection profile are then argued as evidence in favor of the so-called locked-moment model. Within this model, the cluster spin is treated as linked to a cluster crystallographic axis (see Ref. [7], and references therein). Other models treat the spin-relaxation problem by considering cluster rotations $[12,13]$ and the related stochastic processes [14]. While we do not discard the lockedmoment model that gives rise to the broad and welldeveloped deflection profile and, in particular, gives the long tail for the large deflections, observed with a number of clusters including 17 and $22 \mathrm{Gd}$ atom $\left(\mathrm{Gd}_{17}\right.$ and $\left.\mathrm{Gd}_{22}\right)$ clusters, discussed in detail herein, we do discuss other mechanisms for the asymmetric deflection profile formation.

We consider the previously neglected [7-11] mass distribution (that occurs even for a specific cluster size of gadolinium atoms) and the existence of a velocity distribution for an ensemble of clusters. This mass distribution is a consequence of seven naturally occurring gadolinium isotopes. Before adding complexities, associated with moment orientation, to the deflection of a cluster in a Stern-Gerlach experiment, it seems expedient that any theory aimed at describing the deflection profile must begin with the classical formula that describes the deflection as a function of the mass of the cluster $m$, the cluster velocity $v$, the cluster magnetization $M_{z}$, the gradient field $(d H / d z)$, the length of the gradient field region, $L$, and the distance from the gradient magnet to the ionization region, $D$ :

$$
z=\frac{M_{z}(d H / d z)\left(D L+L^{2} / 2\right)}{m v^{2}} \equiv \frac{K}{m v^{2}} .
$$




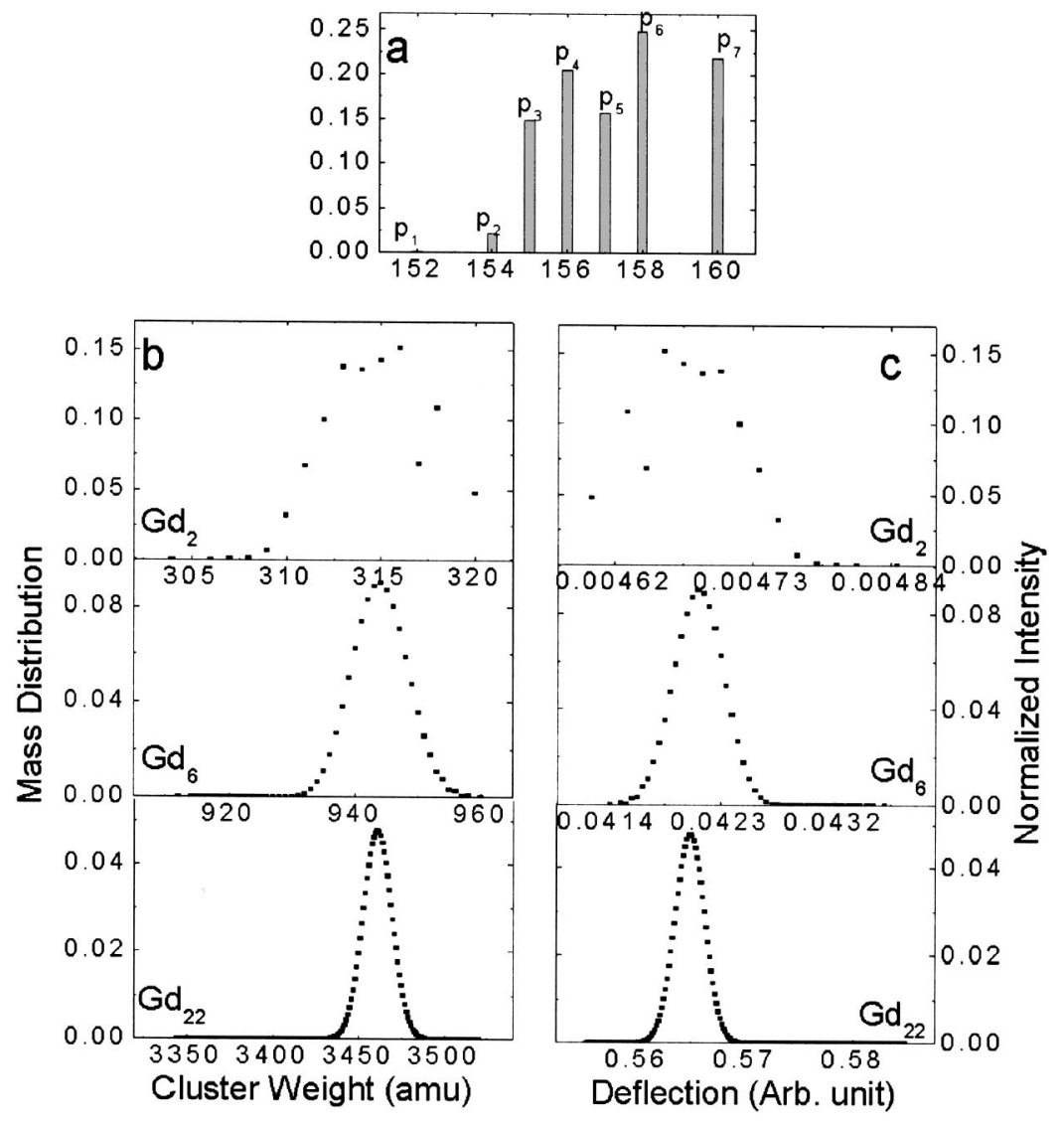

FIG. 1. On the top (a), the percentages of the naturally occurring seven gadolinium isotopes; on the left (b), the mass distributions for $\mathrm{Gd}_{2}$, $\mathrm{Gd}_{6}$, and $\mathrm{Gd}_{22}$ clusters as a result of the combining different natural isotopes; and on the right (c) the deflection profiles for $\mathrm{Gd}_{2}, \mathrm{Gd}_{6}$, and $\mathrm{Gd}_{22}$ clusters due to the mass distributions are shown assuming a single cluster velocity.

This formula contains two parameters which previously were not considered as anything other than single valued: the cluster mass $m$ and the cluster longitudinal velocity $v$. In reality, the experimental methods used in the Stern-Gerlach experiments, to date, do not constrain these parameters to fixed values. This is important as these parameters are in the denominator of Eq. (1); thus the dependence of the deflection, on variations in cluster mass and cluster velocity, are essentially nonlinear. Even in case of a symmetric mass distribution, with respect to some average magnitude of the cluster mass, the resulting deflection profile will be, necessarily, asymmetric. Similarly, the resulting deflection profile will also be asymmetric with a finite (non-single-valued) but symmetric velocity distribution.

\section{THE GADOLINIUM CLUSTER MASS DISTRIBUTION}

We note that the naturally abundant Gd occurs as several isotopes. The possible different combination of $\mathrm{Gd}$ atoms of different isotopes dictates that, even for a fixed number of atoms in the cluster, a Gd cluster can exhibit a range of masses. As a consequence, in accordance with Eq. (1), the range of masses exhibited by an ensemble of clusters, of a fixed number of atoms $n$, will necessarily give rise to the deflection profile of a finite width and certain shape rather than a single magnitude of deflection. While we show that for sufficiently big cluster sizes, the resulting mass distribution is reduced to the Gaussian-like distribution that is symmetric with respect to the average magnitude of the cluster mass, nonetheless, the resulting deflection profile is asym- metric and the asymmetry obtained corresponds to the type (although a smaller magnitude) of the asymmetry obtained in the modern Stern-Gerlach experiment. The percentages of the seven naturally occurring gadolinium isotopes (indexed as $i=1,2, \ldots, 7)$ are denoted by $P_{i}$ and shown in Fig. 1(a). In a given " $r$ " numbered $\mathrm{Gd}_{r}$ cluster, the $r$ atoms are likewise composed of the various isotopes. The different possible combinations result in a distribution of masses. If the num-

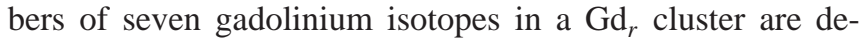
noted by $k_{i}$, with $i=1,2, \ldots, 7$, we have the following relationship:

$$
\sum_{i=0}^{7} k_{i}=r
$$

For any combination of $k_{i=1,2, \ldots, 7}$ satisfying Eq. (2), the probability for this can be given by

$$
P_{r}\left(k_{1}, k_{2}, \ldots, k_{7}\right)=\frac{r !}{k_{1} ! k_{2} ! \ldots k_{7} !} p_{1}^{k_{1}} p_{2}^{k_{2}} \ldots p_{7}^{k_{7}}
$$

The mass of the $\mathrm{Gd}_{r}$ cluster is defined by

$$
m=\sum_{i=0}^{7} k_{i} m_{i}
$$

where $m_{i}$ represents the mass of isotope $i$. Generally, a possible mass $m$ of a $\mathrm{Gd}_{r}$ cluster can be created by several 
combinations of $k_{i}$ satisfying both Eqs. (2) and (4). Therefore, the mass distribution of $\mathrm{Gd}_{r}$ clusters can be determined by

$$
P(m)=\sum_{k_{i}} \frac{r !}{k_{1} ! k_{2} ! \cdots k_{7} !} p_{1}^{k_{1}} p_{2}^{k_{2}} \cdots p_{7}^{k_{7}}
$$

The mass distributions for some different gadolinium cluster sizes are shown in Fig. 1(b). In the smallest $\mathrm{Gd}_{2}$ cluster, the mass distribution displays several minima and maxima, a consequence of the distribution of the seven naturally occurring abundant gadolinium isotopes [Fig. 1(a)]. With an increase of cluster size, the mass distribution shows only one maximum and is symmetric about the maximum. Figure 1 clearly shows that Gd clusters have a larger range of masses with a larger number of atoms in the cluster.

\section{THE INFLUENCE OF A FINITE VELOCITY DISTRIBUTION ON DEFLECTION}

The experimentalists' ability to measure the cluster velocity is based on the time interval between the moment when the cluster passes through a slit in a rotating chopper wheel and the moment when it appears in the ionization region. This does not guarantee that the velocity is an absolutely fixed parameter. On the contrary, the velocity has a range of values because the time of flight is determined by a slit of finite (nonzero) width and the time interval for the slit to traverse the cluster beam path that is also nonzero. In addition, the measurement of the total time of flight cannot be perfect. As a consequence, there necessarily exists a finite longitudinal velocity distribution in the cluster beam. Combined with these more prosaic complications is the fact that, during the flight time interval, the cluster could experience collisions with $\mathrm{He}$ atoms and this too can lead to a less-thanideal velocity distribution.

One should distinguish the translational temperature of the cluster beam and the internal vibrational temperature(s) of the clusters. The former determines the longitudinal velocity distribution, whereas the latter influences the magnitude of the cluster magnetization. These two "temperatures" are different because, as the clusters form and pass through the supersonic nozzle, there are differences in the effectiveness of the He atom to cluster cross sections (effectiveness of the He atom collisions on the cluster beam) in cooling of the cluster vibrational and translational degrees of freedom. The translational temperature tends to be lower than the vibrational temperature. The small magnitude of the cluster translational temperature, together with the large cluster mass, results in the narrow width of the longitudinal velocity distribution, and this is the basis for treating the movement of clusters down a Stern-Gerlach experiment with one welldefined magnitude of longitudinal velocity.

We attempt to take into account the existence of a narrow longitudinal velocity distribution. The existence of the velocity distribution must give rise to the existence of a deflection profile of finite width because clusters with different longitudinal velocities spend different times in the region of the gradient magnet and thus deflect to different distances. The dependence of the deflection on the cluster velocity is nonlinear and the resulting deflection profile is also asymmetric. Due to the second power of the velocity in the denominator in Eq. (1), the nonlinear dependence of the deflection profile on velocity is even stronger than the nonlinear deflection dependence due to mass, discussed later.

In order to determine the deflection profiles due to both the mass and velocity distributions, we choose to model $\mathrm{Gd}_{22}$ clusters, in part because these clusters exhibit superparamagnetic behavior [7]. Hence, we do not need to include the complicated ensemble averaged over the initial conditions. This is a simpler situation than that for some other cluster sizes, for example, $\mathrm{Gd}_{17}$ [7]. Thus, the time-averaged magnetic moment in the magnetic field can be determined by the Langevin function [7]:

$$
\bar{\mu}=N \mu[\operatorname{coth}(x)-1 / x],
$$

where $x=N \mu H / k_{B} T, \mu$ is the internal magnetic moment, and $H$ is the magnetic field. $\bar{\mu}$ is used to calculate the total deflection $z$ from

$$
z=\frac{\bar{\mu}(d H / d z)\left(D L+L^{2} / 2\right)}{m v^{2}}=\frac{C}{\varepsilon},
$$

where $C=0.5 \bar{\mu}(d H / d z)\left(D L+L^{2} / 2\right)$ is related to $\bar{\mu}$ and the experimental conditions: the magnetic-field gradient $(d H / d z)$, the length of magnet $L$, and the distance $D$ between the magnet and the detector. $\varepsilon=m v^{2} / 2$ is the translational energy of the clusters. From Eq. (7), only a cluster with the translational energy $\varepsilon$ can reach a point $z$ on the screen (detector).

Since the cluster beam has essentially one-dimensional movement, we model the longitudinal velocity distribution by the one-dimensional Maxwell-Boltzmann distribution that is just the Gaussian function:

$$
\varphi(v)=n \sqrt{\frac{m}{2 \pi k_{B} T_{\mathrm{tr}}}} \exp \left\{-\frac{m(v-u)^{2}}{2 k_{B} T_{\mathrm{tr}}}\right\},
$$

where $T_{\text {tr }}$ is the internal translational temperature of the cluster beam and $u$ is the average velocity of clusters in the beam defined by the "measured" time-of-flight parameter in the Stern-Gerlach experiment. Here, we treat the mass of the cluster as constant (although this was just shown to be over simplistic).

To find the effect of longitudinal velocity distribution on the deflection profile in the Stern-Gerlach experiment, one should again use Eq. (1), which establishes the relationship between the velocity of the cluster and the resulting deviation. The corresponding relationship between a positive deflection interval $d z$ and the positive velocity interval $d v$ is determined by $d z=2 C d v / m v^{3}$. From the Eq. (8), the number of clusters whose velocity belongs in the interval $(v, v$ $+d v$ ) is equal to $d n=n \varphi(v) d v$. With the help of these two results, one can obtain the part of the whole cluster ensemble whose deflections belong in the interval $(z, z+d z)$. 


$$
\begin{aligned}
I(z) & \equiv \frac{d n}{n d z}=\frac{1}{2 \bar{z} w \sqrt{\pi}} \exp \left\{-\frac{1}{w^{2}}\left(\sqrt{\frac{\bar{z}}{z}-1}\right)^{2}\right\}\left(\frac{\bar{z}}{z}\right)^{3 / 2} \\
\equiv & \frac{I(x)}{\bar{z}} \\
I(x) & =\frac{1}{2 w \sqrt{\pi}} \exp \left\{-\frac{1}{w^{2}}\left(\frac{1}{\sqrt{x}}-1\right)^{2}\right\} \frac{1}{x^{3 / 2}} \\
w & =\sqrt{\frac{2 k_{B} T_{\mathrm{tr}}}{m u^{2}}}, \quad \bar{z}=\frac{2 C}{m u^{2}}, \quad x=\frac{z}{\bar{z}}
\end{aligned}
$$

Note that $I(x)$ is a dimensionless parameter and thus it can be treated as a dispersion only for the distribution with respect to the magnitudes of the dimensionless parameter $x$. The magnitude of the effective dispersion $I(z)$, corresponding to the distribution in the real $z$ space, can be evaluated in the following way. Part of the expression in Eq. (9.2) can be expanded in the vicinity of the point $x=1$ that corresponds, with a high degree of accuracy, to the maximum magnitude of $I: 1 / \sqrt{x}-1 \approx-1 / 2(x-1)$, and thus the whole argument in the exponent will appear as

$$
-\frac{(x-1)^{2}}{4 w^{2}} \equiv-\frac{(z-\bar{z})^{2}}{4 w^{2} \bar{z}^{2}} \equiv-\frac{(z-\bar{z})^{2}}{w_{z}^{2}} .
$$

The expression for the $z$ dispersion is then determined by the parameter $w_{z}=2 w \bar{z}$. Therefore, the expression for $I(z)$ may be rewritten as

$$
I(z)=\frac{1}{w_{z} \sqrt{\pi}} \exp \left\{-\frac{(z-\bar{z})^{2}}{w_{z}^{2}}\right\}\left(\frac{\bar{z}}{z}\right)^{3 / 2} .
$$

One should realize that the expansion we used in the process of the evaluation of this formula is valid only in the vicinity of $x=1$, or equivalently, in the vicinity of the point $z=\bar{z}$. For this reason, one should apply Eq. (10) only in the same region. Compared to the exact result, provided by Eqs. (9), Eq. (10) gives rise to an infinite magnitude of $I$ in the vicinity of the point $z=0$ and results in the wrong kind of asymmetry. Equation (10) serves only as a starting point for the evaluation of the important characteristic of the $z$ dispersion, $w_{z}=2 w \bar{z}$, that determines the width of the deflection profile in the $z$-space direction. Due to the dependence of the average deflection $\bar{z}$ on magnetic field $H$, and the vibrational temperature $T_{\text {vib }}$, the width of the deflection profile depends on other parameters as well. Since the increase in the external field leads to the increase in the average deflection $\bar{z}$, then it follows from $w_{z}=2 w \bar{z}$ that the deflection profile width must increase too. This is exactly what was observed in the experiment with the superparamagnetic cluster $\mathrm{Gd}_{22}$ and $\mathrm{Co}_{120-140}$ clusters (e.g., Figs. 1(a) and 1(c) in Ref. [15]).

Bearing in mind that one of our main goals is to investigate the asymmetry in the deflection profile resulting from the longitudinal velocity distribution, we plotted $I(z)$ versus $z$ for different half-widths of $w_{z}$, and this is provided in Fig. 2. It can be seen that the plot of $I(z)$ is almost symmetric for

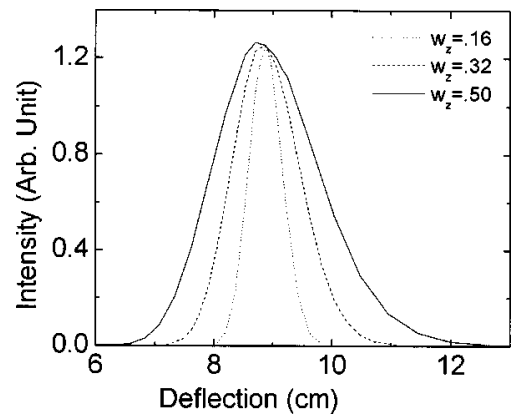

FIG. 2. The calculated deflection profile for $\mathrm{Gd}_{22}$ clusters at several half-width values of the velocity distribution and nominal velocity of $v_{0}=10 \mathrm{~m} / \mathrm{s}$.

small values of the half-width $w_{z}$. As the value of the halfwidth $w_{z}$ increases, the asymmetry of $I(z)$ becomes more apparent. Thus, the degree of the asymmetry of the deflection profile is determined by the value of the half-width $w_{z}$, which is, in turn, determined by the translational velocity $v$, the cluster mass $m$, and the translational temperature $T_{\mathrm{tr}}$. It follows from Eq. (9.3), the smaller the cluster mass and the higher the temperature $T_{\mathrm{tr}}$, the larger the degree of asymmetry. Therefore, the transition-metal clusters must exhibit more asymmetric deflection profiles than the rare-earth clusters of the same size, because the mass of transition-metal atoms is approximately three times smaller. Since the experimental data available contain results for small rare-earth clusters $(N=20-30)$ and large transition-metal clusters $(N$ $=120-140$ ), comparison of this postulated theoretical trend with experiment is difficult.

In the approach considered above, the degree of the asymmetry of the deflection profile does not change with external field. We conclude that we can treat the visible increase in the degree of the asymmetry observed in Ref. [15] as a consequence of the change in scaling. In the absence of an external field, the deflection profile $I(z)$, Eq. (9.1), transforms into the $\delta$ function with zero width, and is thus symmetric (there is no deflection regardless of the magnitude of its longitudinal velocity). As soon as any nonzero magnitude of the field is applied, the width of the deflection profile becomes nonzero and thus the asymmetry, determined by Eqs. (9.2) and (9.3), becomes increasingly evident. Further increases in the external field leads to the increase in the width of the deflection profile, determined by the $z$ dispersion $w_{z}=2 w \bar{z}$.

We realize that modeling the velocity distribution function by the one-dimensional Maxwell-Boltzmann distribution, Eq. (8), with definite translational temperature $T_{\text {tr }}$ is a substantial simplification. The real velocity distribution of clusters passing through the supersonic nozzle is an equilibrium distribution (as discussed further below) and thus the very concept of the translational temperature is not applicable. Since we do not know the real velocity distribution function, the evaluation based on the "real" velocity distribution is beyond the scope of this present effort. We also realize that the use of a simplified velocity distribution places restrictions on our conclusions. 


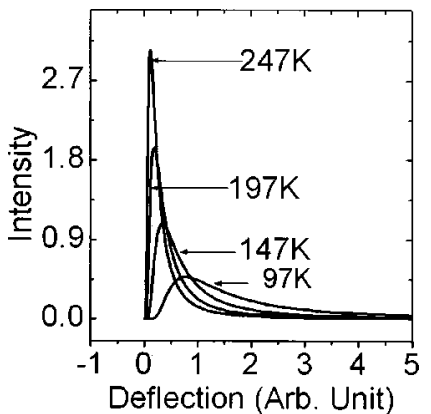

FIG. 3. The calculated deflection profiles for $\mathrm{Gd}_{22}$ clusters at several temperatures.

\section{THE INFLUENCE OF A FINITE MASS DISTRIBUTION ON DEFLECTION}

Though there is a mass distribution for each cluster size [as seen in Fig. 1(b)], when trying to model the end deflection profile, this mass distribution is subsumed into the translational energy $\varepsilon$, so that we can assume that an ensemble of $\mathrm{Gd}_{22}$ clusters follows the probability distribution for $\varepsilon$ :

$$
f(\varepsilon) d \varepsilon=\frac{2 \pi}{(2 \pi k T)^{3 / 2}} \sqrt{\varepsilon} \exp (-\varepsilon / k T) d \varepsilon,
$$

where $f(\varepsilon) d \varepsilon$ represents the probability that a cluster has the translation energy in the range $\varepsilon$ to $\varepsilon+d \varepsilon$. The combination of Eqs. (6) and (7) gives the total probability that a cluster appears at a given deflection $z$ to $z+d z$ :

$$
P(z)=\frac{2}{\sqrt{\pi}}\left(\frac{C}{k T}\right)^{-1}\left(\frac{C}{k T z}\right)^{5 / 2} \exp (-C / k T z) d z
$$

From Eq. (12), it is difficult to infer the extent of the effect of the cluster mass distribution alone on the deflection profile. Nonetheless, from the mass distributions in Fig. 1(a), we can provide a good estimate of this effect by assuming that all the Gd clusters have the same velocity (corresponding to using the "perfect" time-of-flight chopper in the experiment) and then calculate the deflection profiles due to the mass distribution. The results are shown in Fig. 1(c). Except for the smallest $\mathrm{Gd}_{2}$ cluster, the deflection profiles have the same shape as the mass distribution. By increasing the cluster size, the range of the mass distribution becomes broader, and then the range in which the clusters appear on the screen become broader. Hence, for larger clusters, the mass distributions may need to be considered to correctly model the experimental deflection profiles, particularly for very narrow velocity distributions. The mass distribution alone, however, cannot explain experimental data with a tailing distribution to large deflections, particularly for the larger clusters.

\section{THE POSSIBILITY OF A NONEQUILIBRIUM TEMPERATURE DISTRIBUTION}

In Fig. 3, we show the calculated deflection profiles from Eq. (12) for $\mathrm{Gd}_{22}$ as a function of temperature. By decreasing the temperature, the calculated profiles due to the translation energy distribution become broader. The most interesting

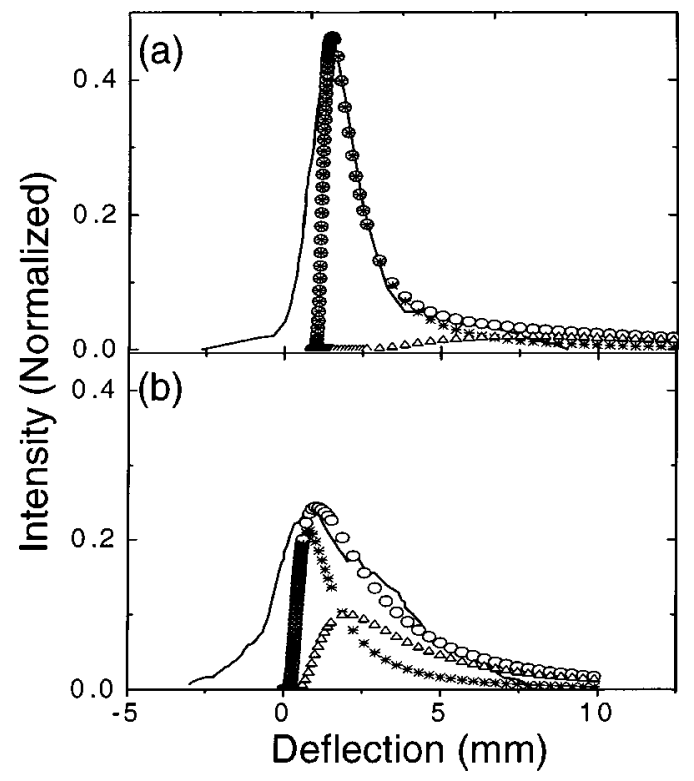

FIG. 4. The experimental and the calculated deflection profiles for (a) $\mathrm{Gd}_{22}$ and (b) $\mathrm{Gd}_{17}$. The experimental deflection profile from Ref. [7] are shown as a solid curve, and fitted to a variety of calculated translational temperatures. For $\mathrm{Gd}_{22}$ (a), the calculated profile for $115 \mathrm{~K}$ (at $70 \%$ of the cluster intensity) is shown by $*$ and the calculated profile for $35 \mathrm{~K}$ (at $30 \%$ of the cluster intensity) is indicated by $\triangle$. For $\mathrm{Gd}_{17}$ (b), the calculated profile for $97 \mathrm{~K}$ (at $45 \%$ of the cluster intensity) is shown by $*$ and the calculated profile for 60 $\mathrm{K}$ (at $55 \%$ of the cluster intensity) is indicated by $\triangle$. The sum of the calculated profiles at $97 \mathrm{~K}$ and $60 \mathrm{~K}$ are shown as $(\bigcirc)$. Inclusion of a broader initial velocity distribution and the mass distribution improve the "fit."

feature is the long tail for the largest deflections (to the right side) of the profiles, especially at low temperatures. In Fig. 4, the solid curve gives the Stern-Gerlach experimental deflection profile for a nominal vibrational temperature of $97 \mathrm{~K}$ for $\mathrm{Gd}_{17}$ and $\mathrm{Gd}_{22}$ taken from Ref. [7]. The shapes of deflection profile are not symmetric. In Ref. [7], it was assumed that the clusters reached equilibrium before they entered the magnet. The mass distribution (as seen from Fig. 1) and a small velocity distribution (as seen from Fig. 2) are by themselves, or even in combination, insufficient to explain this experimental deflection profile.

We cannot fit the experimental data easily assuming a single translational temperature. To obtain the calculated profiles with the best fit to experiment, indeed to best explain the large tail to high deflections in the experimental data, we are required to add profiles for two or more translational temperatures. In Fig. 4, we compare calculated profiles for two temperatures to the experimental data for $\mathrm{Gd}_{17}$ and $\mathrm{Gd}_{22}$. In this model, we assume that $70 \%$ of the $\mathrm{Gd}_{22}$ clusters have a translational temperature of $115 \mathrm{~K}$ and $30 \%$ have a translational temperature of $35 \mathrm{~K}$. For $\mathrm{Gd}_{17}$, we have fit the experimental data assuming that $45 \%$ of the $\mathrm{Gd}_{17}$ clusters have a translational temperature of $97 \mathrm{~K}$ and $55 \%$ have a translational temperature of $60 \mathrm{~K}$.

These are not unique fits to the data, but are far better fits than any single temperature. The combinations of mass, velocity, and temperature distributions can combine in a variety 
of ways to cause the long tail on the right side (higher deflections) of the deflection profiles at low temperature. It is clear that consideration of the distribution of translation energy and temperature are not enough to explain the experimental profiles alone, but the calculated profile gives a good fitting to the experimental tail at high deflection (on the right side of the curves in Fig. 4).

We suggest that the clusters in this Stern-Gerlach experiment do not have the same vibrational and translational temperatures, but rather represent an inhomogeneous distribution in both vibrational and translational temperatures. The reason may be that at low temperatures, very cold clusters are suppressed in the experiment. In this way, the long tail on the right side of the profiles may be produced by the high translation energy clusters. This may be a consequence of inhomogeneous cooling processes as the clusters form, possibly resulting from two or more competing cooling mechanisms: sublimation of daughter fragments and inelastic collisions, for example.

\section{CONCLUSIONS}

To summarize, we considered the effects of the cluster mass and the longitudinal velocity distribution on the deflection profile. There exists a mass distribution in Gd clusters because of the numerous naturally occurring $\mathrm{Gd}$ isotopes. For gadolinium clusters of small size, the modification on the deflection profile due to the mass distribution is so small that it can be neglected, as has generally been the approach in the past. Due to the nonlinear inverse dependence of the deflection on the cluster mass and the longitudinal velocity, the resulting deflection profiles exhibit asymmetry, as observed in the recent experiments with some rare-earth metal clusters, even in case of the symmetric mass and velocity distributions [15]. We find that the mass distribution has almost negligible effect on the degree of the asymmetry of the resulting deflection profile, whereas the longitudinal velocity distribution gives rise to more asymmetry in the deflection profiles. More realistic velocity distributions are needed for the detailed comparison of theory with experiment. Upon closer inspection of the experimental data, the translation energy plus the vibrational temperature distribution may be inhomogeneous and not uniform. This could be the reason that there is a long tail at high deflections of the experimental deflection profiles at low temperature in the magnetic cluster Stern-Gerlach experiments.

\section{ACKNOWLEDGMENTS}

This work was supported by the National Science Foundation (NSF) through Grant No. DMR-98-02126, the Center for Materials Research and Analysis (CMRA), the Nebraska Research Initiative at the University of Nebraska, and the North Atlantic Treaty Organization (NATO) under Grant No. PST.CLG.976845.
[1] P. A. Dowben, Surf. Sci. Rep. 40, 151 (2000).

[2] D. M. Cox, D. J. Trevor, R. L. Whetten, E. A. Rohlfing, and A. Kaldor, Phys. Rev. B 32, 7290 (1985).

[3] D. M. Cox, R. L. Whetten, E. A. Rohlfing, and A. Kaldaor, J. Chem. Phys. 84, 4651 (1985).

[4] W. A. de Heer, P. Milani, and A. Chatelain, Phys. Rev. Lett. 65, 488 (1990).

[5] J. P. Bucher, D. C. Douglass, P. Xia, B. Haynes, and L. A. Bloomfield, Z. Phys. D: At., Mol. Clusters 19, 251 (1991).

[6] J. P. Bucher, D. C. Douglass, and L. A. Bloomfield, Phys. Rev. Lett. 66, 3052 (1993).

[7] D. C. Douglass, A. J. Cox, J. P. Bucher, and L. A. Bloomfield, Phys. Rev. B 47, 12874 (1993).

[8] L. A. Bloomfield, J. Deng, A. J. Cox, J. W. Emmert, F. K. Fatemi, H. Zhang, D. B. Haynes, J. G. Louderback, D. C. Douglass, J. P. Bucher, and A. M. Spencer, in Magnetism and
Electronic Correlations in Local-Moment Systems: Rare-Earth Elements and Compounds, edited by M. Donath, P. A. Dowben, and W. Nolting (World Scientific, Singapore, 1998).

[9] S. N. Khanna and S. Linderoth, Phys. Rev. Lett. 67, 742 (1991).

[10] G. F. Bertsch and K. Yabana, Phys. Rev. B 56, 3222 (1997).

[11] G. F. Bertsch, N. Onishi, and K. Yabana, Z. Phys. D: At., Mol. Clusters 34, 213 (1995).

[12] V. Visuthikraisee and G. F. Bertsch, Phys. Rev. A 54, 5104 (1996).

[13] G. F. Birtch and K. Yabana, Phys. Rev. A 49, 1930 (1994).

[14] S. Dattagupta and S. D. Mahanti, Phys. Rev. B 47, 10244 (1998).

[15] D. Gerion, A. Hirt, and A. Chatelain, Phys. Rev. Lett. 83, 532 (1999). 